\section{factor prices in general equilibrium}

\section{Introduction}

At first glance, the Walrasian general equilibrium model does not offer a theory of factor prices. Factors are goods supplied by agents to firms who then use them to produce outputs. In the general equilibrium model, there is no such class of goods: one and the same good can simultaneously be used as an input by some firms, produced as an output by other firms, sold by some consumers, and purchased and consumed by other consumers. Indeed, the general equilibrium model's abstraction from the minutiae of how particular goods are used is one of the theory's great advantages. For many of the classical concerns of the Walrasian tradition - the existence of equilibrium, optimality - these details are irrelevant.

Even if there is a category of factors that consumers sell and firms buy, it is hard to see any distinctive properties of these goods. While factor supply functions can exhibit perverse responses to price changes, so can output demand functions. The responses of firms to price changes are better behaved, and firm factor demands may seem to be governed by a distinctive principle: a firm's demand for a factor diminishes in its own price while a firm's supply of an output increases in its own price. While correct, these two fundamental rules of producer comparative statics are really reflections of a single law, as Samuelson (1947) showed long ago. Suppose in an $\ell$-good economy that a profit-maximizing firm with production set $Y \subset R^{\ell}$ chooses $y=\left(y_{1}, \ldots, y_{\ell}\right) \in Y$ when facing prices $p=\left(p_{1}, \ldots, p_{\ell}\right)$ and $\hat{y} \in Y$ when facing $\hat{p}$. Since each decision is profit-maximizing, $p \cdot y \geq$ $p \cdot \hat{y}$ and $\hat{p} \cdot \hat{y} \geq \hat{p} \cdot y$ and hence $(\hat{p}-p) \cdot(\hat{y}-y) \geq 0$. If only one price differs at $p$ compared to $\hat{p}$, say the first, then $\left(\hat{p}_{1}-p_{1}\right)\left(\hat{y}_{1}-y_{1}\right) \geq 0$. So if $\hat{p}_{1}>p_{1}$ then $\hat{y}_{1} \geq y_{1}$. Both of the comparative statics rules now follow from the appropriate sign restrictions on $y_{1}$ and $\hat{y}_{1}$ : when both are positive we conclude that the output of good 1 supplied by the firm must be weakly increasing in its price, while if both are negative we conclude that the factor demand for good 1 must be weakly decreasing in its price (since $\hat{y}_{1} \geq$ $y_{1}$ and $\left(\hat{y}_{1}, y_{1}\right) \leq 0$ imply $\left.\left|\hat{y}_{1}\right| \leq\left|y_{1}\right|\right)$. It is tempting to conclude that there is no special general equilibrium principle of factor demands, just a specific application that follows when the sign convention for factors is inserted.

\section{Factor-price indeterminacy}

The demand for and supply of factors can nevertheless exhibit distinctive properties, although they are consistent with the generalities pointed out in the previous section. These properties do not matter for the most of the classical results of general equilibrium theory, but they can undermine one result, the generic determinacy (local uniqueness) of equilibria.
The first distinguishing trait of factors is that sometimes they do not provide any direct utility and are useful only as inputs in production. Consumers will supply to the market their entire endowment of such 'pure' factors and hence supply will be inelastic with respect to price changes. As we will see, what matters is local unresponsiveness to prices. Perhaps when a factor such as iron ore is sufficiently cheap in terms of consumption goods consumers will find some direct use for it and hence have an excess demand that locally varies as a function of prices. But above some minimum price, consumers will not consume any iron ore and in this range consumers' excess demand will be inelastic. Second, technology can restrict the number of ways in which factors can be productively combined. The extreme case occurs with fixed coefficients - the Leontiev production function - where to produce one unit of a good just one combination of factors will do. More flexible is the linear activities model where finitely many constant-return-to-scale techniques are available to produce one or more goods. Factors then may be combined in various configurations but some factor proportions cannot be used productively (that is, without disposing of some of the factors). Nonlinear activities are qualitatively similar but do not require constant returns to scale. In all these cases, production sets have a kinked rather than smooth (differentiable) surface. Consequently factor prices can be adjusted at least slightly from one equilibrium configuration without changing the quantity of factors that profit-maximizing producers will demand when producing a given quantity of output (or vector of outputs). In the Leontiev case, picture the multiple price lines that can support the model's L-shaped isoquants. Of course, production sets do not have to exhibit kinks; for example, they will be smooth when each output is a differentiable function of factor inputs. Any change in relative factor prices will then lead to a change in factor demand.

Factors of production thus are distinctive in that both demand and supply can be unresponsive to certain types of price changes. Factor demand and supply do not have to display this unresponsiveness, but under plausible circumstances permitted by the general equilibrium model they will. Inelastic factor demand and supply in turn can lead to an indeterminacy of factor prices. For a simple example, suppose an economy has one consumption good, produced by a single linear activity that requires $a_{1}$ units of one factor and $a_{2}$ units of a second factor to yield one unit of output. Set the price of consumption equal to 1 , let $w_{1}$ and $w_{2}$ be the two factor prices, let the endowments of the two inelastically supplied factors be $e_{1} \geq 0$ and $e_{2} \geq 0$, and let $y$ be the sole activity usage level. An equilibrium $\left(w_{1}, w_{2}, y\right) \geq 0$ where the consumption good is produced and has a positive price must satisfy three conditions: (i) $a_{1} w_{1}+a_{2} w_{2}=1$ (the activity breaks even), (ii) $a_{i} y \leq e_{i}$ for $i=1,2$ (market-clearing for factors), and (iii) $a_{i} y<e_{i} \Rightarrow w_{i}=0$ for $i=1,2$ (factors in excess supply have a 0 price). On the assumption that the demand for 
output equals factor income, which is a form of Walras' law, (i)-(iii) imply that the market for output clears. Evidently equilibrium must satisfy $y=\min \left[e_{1} / a_{1}, e_{2} / a_{2}\right]$. By (iii), the two factors will both have a strictly positive price only if

$$
\frac{e_{1}}{a_{1}}=\frac{e_{2}}{a_{2}},
$$

in which case any $w=\left(w_{1}, w_{2}\right) \geq 0$ that satisfies (i) will be an equilibrium $w$ : indeterminacy therefore obtains when (1) holds. We defer for a little while the question of whether this knife-edge condition is likely to be satisfied.

Fixed coefficients and inelastic factor supply do not always lead to indeterminate factor prices. Prior to the invention of the differentiable production function and for awhile thereafter, the standard cure for factor-price indeterminacy was to argue that, even if each industry uses factors in fixed proportions, those proportions will differ across industries; variations in factor prices will then lead to changes in relative output prices, and thus to changes in output demand that feedback to changes in factor demand (Cassel, 1924; Wieser, 1927). Substitution in consumption can thereby play the same equilibrating role as the technological substitution of inputs in production. For the simplest example, suppose we supplement the above single-sector economy with a new sector that uses $b_{1}$ units of the first factor and $b_{2}$ units of the second factor to produce one unit of a second consumption good. If we keep the price of the first consumption good equal to 1 , and let $p_{b}$ be the price of the second consumption good, then when both activities break even the equalities

$$
a_{1} w_{1}+a_{2} w_{2}=1, \quad b_{1} w_{1}+b_{2} w_{2}=p_{b}
$$

must be satisfied. As long as $a_{1} / a_{2} \neq b_{1} / b_{2}$, it will not be possible to adjust $w$ without also changing the relative price of the consumption goods $p_{b}$. When $w_{1} / w_{2}$ increases, the consumption good that uses factor 1 more intensively will rise in price, presumably diminishing demand for that good and thus diminishing the demand for factor 1. Even if demand for consumption is a perverse function of prices, this two output-two factor model will still typically have determinate prices as long as both activities break even.

A general linear activity analysis model will clarify when the determinate and indeterminate cases arise. The linearity of the activities serves only to simplify the model's equilibrium conditions. There will be two types of goods: factors, which give no utility and are inelastically supplied, and consumption goods, which do give utility. Despite their name, consumption goods can be used as inputs and nonproducible but they must provide utility to some agents. We now adopt the standard sign convention and define an activity to be a vector, with as many coordinates as there are commodities, whose positive coordinates give the quantities of goods produced and negative coordinates give the quantities of goods used when the activity is operated at the unit level. In equilibrium the excess demand for each good must be non-positive, each good in excess supply must have a 0 price, each activity must earn non-positive profits, and each activity in use must earn 0 profits. Since determinacy and indeterminacy are purely local events, a search for equilibrium prices and activity near a reference equilibrium can ignore the 'slack' equilibrium conditions, the market-clearing condition for any good in excess supply and the no-positive-profits condition for any activity that either makes strictly negative profits or utilizes and produces only goods in excess supply: for small adjustments of prices and activity levels, the excluded goods will remain in excess supply and the excluded activities will continue to make negative profits or continue to use and produce only goods in excess supply (and hence continue to break even). Call any good not in excess supply and any activity that breaks even and that uses or produces at least one good not in excess supply 'operative'. Given some reference equilibrium with $\ell$ operative consumption goods, $m$ operative factors, and $n$ operative activities, let $A$ be the $(\ell+m) \times n$ activity analysis matrix whose rows and columns correspond to the operative goods and activities, let $y$ be the $n$-vector of operative activity levels, let $p$ be the $\ell$-vector of prices for the operative consumption goods, let $w$ be the $m$-vector of prices for the operative factors, let $z(p, w)$ be the excess demand function for the operative consumption goods, which we assume is homogeneous of degree 0 in $(p, w)$, and finally let $e$ be the $m$-vector of inelastic supplies of the operative factors. Walras' law then states that $p \cdot z(p, w)=w \cdot e$. Equilibria $(p, w, y) \geq 0$ are locally characterized by the equalities

$$
(z(p, w),-e)=A y, \quad(p, w)^{\prime} A=0 .
$$

(All vectors are column vectors and ' denotes transposition.) Bear in mind that the market-clearing and nopositive-profit inequalities excluded from (2) vary by equilibrium; the activities and goods operative in one equilibrium need not be operative in another. We assume henceforth that, at any equilibirum, each of the operative activities is used at a strictly positive level and that each operative good has a strictly positive price, $(p, w, y) \gg 0$. As usual, the homogeneity of demand allows us to set one of the positively priced goods to be the numéraire and Walras' law implies that one of market-clearing conditions is redundant. So we set the price of the first consumption good not in excess supply to equal 1 and put aside the market-clearing condition for this good. Letting $\bar{z}(p, w)$ denote $z(p, w)$ without the first coordinate, $\bar{A}$ denote $A$ without the first row, and $\bar{p}$ denote $p$ with the first coordinate set equal to $1,(2)$ can be written

$$
\begin{aligned}
& (\bar{z}(\bar{p}, w),-e)=\bar{A} y \\
& (\bar{p}, w)^{\prime} A=0 .
\end{aligned}
$$


Any small change in $(\bar{p}, w, y)$ that satisfies (3)-(4) will then continue be an equilibrium: the variables $(\bar{p}, w, y)$ will remain positive, all excluded goods will remain in excess supply, and all excluded activities will continue to make negative profits or continue to use and produce only goods in excess supply.

The most conspicuous case of factor-price indeterminacy occurs when $m>n$, that is, when there are more operative factors than operative activities. If, beginning at some reference equilibrium, we fix $y$ at its equilibrium value, then as $(\bar{p}, w)$ varies the market-clearing conditions for factors in (3) will continue to be satisfied. But the remaining equilibrium conditions - (4) and the market-clearing conditions for consumption goods in (3) - comprise $n+\ell-1$ equations in the $\ell-1+m$ variables $(\bar{p}, w)$. Hence, if $m>n$ and as long these remaining equilibrium conditions satisfy a rank condition, which allows the implicit function theorem to be applied, indeterminacy will occur. The economy considered earlier where two factors are used by one activity qualifies as an example of the $m>n$ type of indeterminacy, while the economy where two factors are used to produce two goods does not.

A slight variation of this argument applies to a subset of factors. Suppose that $\hat{m}$ of the $m$ operative factors are used by only $\hat{n}$ of the $n$ operative activities, and that $\hat{m}>\hat{n}$. Thus the remaining $n-\hat{n}$ operative activities have 0 entries in the rows of $A$ that correspond to these $\hat{m}$ factors. If we fix the $\hat{n}$ coordinates of $y$ for the activities that do use these $\hat{m}$ factors, then, as the remaining endogenous variables (the other $n-\hat{n}$ activity levels, $\bar{p}$, and $w$ ) change, the market-clearing conditions for the $\hat{m}$ factors will continue to be satisfied. Moreover, the number of remaining endogenous variables is $n-\hat{n}+$ $\ell-1+m$ while the number of remaining equilibrium conditions is $\ell-1+m-\hat{m}+n$. The difference between the number of remaining variables and remaining equilibrium conditions is therefore $\hat{m}-\hat{n}$ and so there are more variables than equilibrium conditions. Indeterminacy therefore obtains (again, given a rank condition).

Factor-price indeterminacy, whether for an economy as a whole or for a subset of an economy's factors, depends critically on production sets that exhibit kinks. By fixing a set of activity levels, the above indeterminacy argument fixes a vector of factor demands and finds a multiplicity of prices at which firms will demand exactly those quantities. If the aggregate production set were smooth, a fixed vector of firm factor demands would be supported by only one vector of relative factor prices.

Factor-price indeterminacy brings dramatic behavioral consequences: agents have a strong incentive to manipulate factor prices and hence markets cannot function competitively. In the two factor-one activity example, where the endowments satisfy (1), the tiniest withdrawal of either factor $i=1$ or $i=2$ from the market will lead the other factor to be in excess supply and have price 0 and hence cause factor $i$ 's price to jump to $1 / a_{i}$.
No matter how small an owner of factor $i$ is as a proportion of the market, it will be in his or her interest to remove a small amount of $i$ from the market. Agents therefore will not behave like price-takers. When more activities are present, the jump in factor prices need not be as large, but a jump will still occur for an arbitrarily small withdrawal of a factor, and hence the incentive to manipulate will remain. The distinctive mathematical feature of factor-price indeterminacy that drives this conclusion is that the equilibrium correspondence fails to be lower hemicontinuous. (The equilibrium correspondence is the correspondence from the parameters of the model, such as the endowments $e$, to the endogenous variables $(\bar{p}, w, y)$.) When the endowments of factors lead to an indeterminate equilibrium, it will usually be impossible at nearby endowment levels to find equilibrium prices near to the prices of the indeterminate equilibrium. Other varieties of indeterminacy in the general equilibrium model, such as the indeterminacy of the overlapping generations model, do not suffer from such a failure of lower hemicontinuity and therefore do not invite market manipulation (see Mandler, 2002).

\section{The emergence of factor-price indeterminacy through time}

We saw in the two factor-one activity example that indeterminacy occurs only if a knife-edge condition on endowments is satisfied. This observation applies to the broader species of factor-price indeterminacy as well. Suppose again that at some reference equilibrium $\hat{m}$ operative factors are used by $\hat{n}<\hat{m}$ operative activities, let $\hat{e}$ be the endowments of these $\hat{m}$ factors, let $\hat{y}$ be the activity levels for the $\hat{n}$ activities, and let $\hat{A}$ be the $\hat{m} \times \hat{n}$ submatrix of $A$ formed by the rows for the $\hat{m}$ factors and the columns for the $\hat{n}$ activities. Then $\hat{A} \hat{y}=\hat{e}$. But since $\hat{A}$ has more rows than columns, for almost every value of $\hat{e}, \hat{A} \hat{y}=\hat{e}$ will have no solution. Hence, for most levels of an economy's endowments, there will be no equilibrium at which $\hat{m}$ operative factors are used by fewer than $\hat{m}$ operative activities. While the failure in these socalled generic cases of the indeterminacy arguments we have given does not show that equilibria are generically locally unique, the literature on regular economies (see in particular Mas-Colell, 1975; 1985; and Kehoe 1980; 1982) has shown that, for generic endowments and preferences, general equilibrium models with linear or nonlinear production activities do have locally unique equilibria.

The determinacy question, however, does not end here. An economy's endowments of produced inputs capital goods - are in any long-term view endogenous variables not parameters. Consequently, even though factor-price indeterminacy does not arise for generic endowments, it is conceivable that those special endowments that lead to indeterminacy will systematically arise 
as the equilibrium activity of an economy unfolds through time. To see that this can indeed happen, we partition an intertemporal economy's dates into two periods, a first period where goods are either consumed or invested in the production of factors and a second period where the factors produced by first-period activities and natural endowments are used to create consumption goods (possibly also with the aid of intermediate inputs produced within the second period). To test whether the nongeneric factor endowments that lead to indeterminacy are likely to appear, we consider intertemporal economies where the endogenous equilibrium production of secondperiod factors leads the total stock of these factors to assume the nongeneric values where indeterminacy arises. If this endogenous second-period indeterminacy obtains for a robust family of equilibria (the equilibria of a nonempty open set of economies), then sequential indeterminacy occurs (Mandler, 1995).

In the Arrow-Debreu view of an intertemporal economy, agents trade just once at the beginning of economic time; after these initial contracts are signed, no further trade occurs, goods are just delivered. To allow for trade at multiple dates, and thus give indeterminacy in later time periods a chance to appear, we assume instead that agents transfer wealth between periods by borrowing or lending assets. Agents then will typically trade every period, and the economies that appear in later periods will have endowments that are endogenously determined by trade in the initial periods. Moreover any indeterminacy of prices in later periods will change the quantities of goods exchanged and hence change agents' utilities. In our setting, with just two periods, we can let the activities that produce second-period factors serve as assets: agents in the first period will buy or sell rights to the outputs of the activities that produce the secondperiod factors and then in the second period receive or deliver the second-period factors they contracted for in the first period and use their income to trade for consumption. The allocation achieved by a two-period Arrow-Debreu intertemporal equilibrium will occur in an equilibrium with two sequential periods of trade if (a) agents in the first period unanimously anticipate a second-period price vector, $(b)$ given those expectations, goods and asset markets in the first period clear, and $(c)$ given asset deliveries, second-period markets clear at the anticipated prices. We omit the routine details of how to decompose an intertemporal equilibrium into a sequential-trading equilibrium (see Radner, 1972) and will just write one equilibrium condition explicitly, the marketclearing equality for second-period factors.

As usual, we consider some reference equilibrium and ignore those goods in excess supply and those activities that make strictly negative profits or that use and produce only goods in excess supply. If there are $k$ operative goods in period 1 , and $\ell$ operative consumption goods and $m$ operative factors in period 2, the activity analysis matrix for the operative goods and activities takes the form

$$
A=\left(\begin{array}{cc}
A_{1} & 0 \\
0 & A_{c 2} \\
A_{f 1} & A_{f 2}
\end{array}\right) \quad \begin{gathered}
k \\
\ell \\
m
\end{gathered}
$$

where the subscript $c$ or $f$ indicates whether the rows are for consumption goods or factors and the subscript 1 or 2 indicates the time activities begin operation. Since presumably the second-period factors are the outputs of time 1 activities and the inputs of time 2 activities, it makes sense to suppose $A_{f 1} \geq 0$ and $A_{f 2} \leq 0$. If we let $y_{i}$ denote the activity levels for operative activities that begin in period $i$ and $e$ the endowment of operative second-period factors, the market-clearing equality for operative second-period factors is

$$
A_{f 1} y_{1}+A_{f 2} y_{2}+e=0 .
$$

In the background lie the remaining equilibrium conditions: market-clearing conditions for excess-supply factors and for all consumption goods, and nonpositive profit conditions for activities.

Consider the restrictions that (5) places on the number of operative factors. If the number of operative activities in the two periods that produce or use the $m$ operative second-period factors is less than $m$, then, for almost every $e$, (5) will have no solution $y=\left(y_{1}, y_{2}\right) \geq 0$. Similarly if there is a subset of $\hat{m}$ operative second-period factors where the number of operative activities in the two periods that produce or use these factors is less than $\hat{m}$, then again (5) will usually have no solution. We may therefore dismiss these cases as unlikely, in line with the literature on regular economies. In the remaining cases, where for each subset of $\hat{m}$ operative second-period factors the number of operative activities in the two periods that produce or use these factors is greater than or equal to $\hat{m}$, then (5) can have a solution $y \geq 0$ for a robust (open) choice of endowment levels $e$. But in these latter cases it could well be that some subset of operative second-period factors - say the entire set of all $m$ of these factors - is used by fewer than $m$ operative second-period activities. For an example, let $m=2$, suppose that the first factor has no endowment but is produced by an activity with factor output coefficient $c_{1}$ while the second factor has a positive endowment in the second period and is not produced. In the second period, both factors are used by one activity with factor usage coefficients $a_{1}$ and $a_{2}$. Then (5) consists of the two equalities

$$
\begin{gathered}
c_{1} y_{1}+a_{1} y_{2}=0, \\
a_{2} y_{2}+e_{2}=0 .
\end{gathered}
$$

Evidently if $a_{1}<0, a_{2}<0, c_{1}>0$, and $e_{2}>0$, then a solution $y \gg 0$ to (6) exists and is robust: for a small variation in the production coefficients or the endowment, a 
solution $y \gg 0$ will continue to exist. In this equilibrium, factor 2 is produced in just the quantity necessary to ensure that neither factor 1 nor factor 2 is in excess supply. For a second example, suppose that factor 2 is produced as well and also has no endowment, and let $y_{1 i}$ denote the usage level of the activity that produces factor $i$. Then (6) is replaced by $c_{1} y_{11}+a_{1} y_{2}=0$ and $c_{2} y_{12}+a_{2} y_{2}=0$. Now efficiency and hence equilibrium will usually require that the two factors are produced in quantities that leave neither in excess supply in period 2; if, say, factor 1 were in excess supply and if $y_{11}$ could be lowered, thereby increasing the output of some firstperiod consumption good, an inefficiency would exist, which is impossible in equilibrium.

Once agents arrive at period 2, they trade again but now the factor outputs produced by the activities that began in period 1 are exogenously given. So in the example given by (6) the endowment of factor 1 in period 2 equals $c_{1} y_{1}$ and one may readily check that this quantity along with $e_{2}$ of factor 2 satisfy the knife-edge condition (1). Thus, despite seeming to be unlikely at a given point in time, the endowments that lead to indeterminacy can endogenously arise.

Intertemporal general equilibrium economies therefore can be sequentially indeterminate. Moreover, factorprice indeterminacy is typically the only source of endogenous indeterminacy. Let us call the equilibria that occur in the later periods of operation of a sequentialtrading equilibrium and that confirm the expectations formed in the initial period 'continuation equilibria'. A continuation equilibrium is indeterminate if it sits amid a continuum of other (usually non-continuation) equilibria.

Sequential indeterminacy theorem (Mandler, 1995). For a generic set of intertemporal economies with linear activities, a continuation equilibrium is indeterminate at some date $t$ if and only if there is a set of $\hat{m}$ operative factors appearing at $t$ or later that are used or produced by fewer than $\hat{m}$ operative activities that begin at $t$ or later.

In contrast, when production sets are smooth, endogenous endowments do not lead to indeterminacy; typically continuation equilibria are locally unique (Mandler, 1997).

\section{Factor price indeterminacy and the hold-up problem}

The endogenous factor-price indeterminacy of the previous section is not an indeterminacy of the equilibria of the entire intertemporal economy or of the corresponding sequential-trading equilibria. As long as the nonproduced endowments of every period of an intertemporal economy avoid certain nongeneric values, and barring flukes in preference or technology coefficients, only a finite number of intertemporal equilibria will exist.
It follows that in a two-period model that displays sequential indeterminacy, almost all of the infinite multiplicity of equilibria of the second-period economy could not form part of a two-period sequential-trading equilibrium: if the prices of almost any of the second-period equilibria were anticipated in period 1 , they would be inconsistent with market clearing. Specifically, if anticipated second-period prices were to vary slightly from the values that hold in a sequential-trading equilibrium, then either assets would no longer share the same rate of return or the common rate of return on assets would change, and hence typically markets would not clear. But bygones are bygones: once period 1 is past, even the second-period equilibria that violate the requirements of an intertemporal equilibrium are equilibria nonetheless when the economy arrives at its second period.

Moreover second-period indeterminacy will prevent sequential-trading equilibria from proceeding smoothly through time: they will be virtually certain to unravel. Since factor prices are indeterminate in the second period, rational agents will predict that an investment in an activity producing a second-period factor will not except by chance earn the rate of return anticipated in the first period of a sequential-trading equilibrium. Investments will therefore differ from their Walrasian levels. The predictions of the general equilibrium model thus become untenable when agents trade repeatedly through time and factor-price indeterminacy is present, even though all the classical presuppositions of the model price-taking agents, no distortions, and so on - obtain.

The inability of second-period markets to ensure that assets earn the rate of return necessary for efficiency amounts to a hold-up problem, but the cause of the problem differs from the conventional diagnosis. In the classical hold-up problem, the owners of two complementary factors Nash bargain over the revenue they jointly earn; hence, if the owner of one of the factors invests to improve the quality of his factor, the owner recoups only a fraction of the increment to revenue, and consequently investment is inefficiently low (Hart, 1995). The problem, it would seem, is that the factor owners form a bilateral monopoly and cannot purchase each other's services on a competitive market. What we have seen, however, is that a hold-up problem can arise with perfectly competitive markets. Even if factor owners can purchase all complementary factors on competitive markets, factor-price indeterminacy can prevent investments in factors from earning the rate of return required in intertemporal equilibrium (and hence the rate necessary for efficiency): an unguided market has no means to select from the continuum of equilibrium factor prices the specific prices that deliver intertemporal efficiency. Factor markets moreover will not operate competitively in the presence of factor-price indeterminacy, which is another cause for the rate of return to deviate from its competitive equilibrium value. For both reasons, the efficient Walrasian levels of investment need not occur. 
Just as in the classical hold-up problem, long-term contracts can mitigate the troubles that factor-price indeterminacy brings. If labour is among the factors in an economy displaying factor-price indeterminacy, then a labour contract may be able to force trading at prices that allow intertemporal efficiency and prevent labourers or capital goods owners from manipulating factor prices by withdrawing their services from the market. Of course, as in the classical hold-up problem, the incompleteness of contracts may hamper the ability of this solution to deliver first-best efficiency. Alternatively, when a set of complementary factors displays factor-price indeterminacy and consists solely of produced goods, then a bundling of the complementary factors in an asset portfolio - that is, in a 'firm' - can eliminate the incentive to manipulate prices. From the vantage point of factor-price indeterminacy, unions and labour contracts and the firm as an institution emerge as devices to enforce competitive equilibria, not as consequences of imperfect competition in factor markets.

\section{Conclusion: factor-price indeterminacy past and present}

Prior to the Arrow-Debreu transformation of generalequilibrium theory, economists were well-aware that linear activities could lead to an indeterminacy of factor prices. The problem was considered from a long-run perspective: a change in a factor price was presumed to persist for many periods, and, although such a change might not lead to an instantaneous change in either the supply or demand for the factor, arguments were deployed for why demand and supply responses would eventually kick in. For example, in response to a wage increase, although existing capital equipment might have fixed labour requirements, newly constructed capital equipment could be built to use labour less intensively. In addition, a wage increase would eventually lead the price of labour-intensive consumption goods to rise, diminishing the demand for these goods and therefore ultimately for labour as well. This effect does not operate immediately since a wage increase will lead to an offsetting fall in the prices of existing stocks of complementary capital inputs. But the prices of newly produced capital inputs are constrained by break-even requirements; hence, given enough time, the prices of labour-intensive consumption goods will increase. (Robertson, 1931, and Hicks, 1932, offered the most detailed long-run theories. See Mandler, 1999, ch. 2.) Although pre-modern explanations of factor prices faced the indeterminacy problem explicitly, and marshalled a rich array of counterarguments for why the problem normally will not be severe, the long-run perspective had its drawbacks: the attention to persistent changes in factor prices masked an inability to explain why factor prices cannot temporarily change. The older long-run theories simply assumed that, in the absence of demand or supply shocks, factor prices will be maintained at their long-run equilibrium values. This presumption amounts to a rudimentary version of the rule that in an intertemporal equilibrium prices should fulfill the expectations that agents formed in earlier periods. As we have seen, the market mechanism will not enforce this rule; a supplementary theory of contracts and institutions is necessary. The Arrow-Debreu treatment of factors (and other goods) at different dates as fully distinct goods naturally raises the question of whether prices can deviate from previously anticipated values even in the absence of shocks, and curiously, therefore, the Arrow-Debreu account of markets points to the need for a theory of non-market institutions. Unfortunately, the Arrow-Debreu tradition also took the model of trading at a single point in time as its benchmark. It is only with the combination of goods rigorously distinguished by date, sequential trading, and production sets with kinks that factor-price indeterminacy will appear.

MICHAEL MANDLER

See also determinacy and indeterminacy of equilibria; general equilibrium; hold-up problem.

\section{Bibliography}

Cassel, G. 1924. The theory of social economy. New York: Harcourt.

Hart, O. 1995. Firms, Contracts, and Financial Structure. New York: Oxford University Press.

Hicks, J. 1932. Marginal productivity and the principle of variation. Economica 25, 79-88.

Kehoe, T. 1980. An index theorem for general equilibrium models with production. Econometrica 48, 1211-32.

Kehoe, T. 1982. Regular production economies. Journal of Mathematical Economics 10, 147-7.

Mandler, M. 1995. Sequential indeterminacy in production economies. Journal of Economic Theory 66, 406-36.

Mandler, M. 1997. Sequential regularity in smooth production economies. Journal of Mathematical Economics 27, 487-504.

Mandler, M. 1999. Dilemmas in Economic Theory. New York: Oxford University Press.

Mandler, M. 2002. Classical and neoclassical indeterminacy in one-shot vs. ongoing equilibria. Metroeconomica 53, 203-22.

Mas-Colell, A. 1975. On the continuity of equilibrium prices in constant returns production economies. Journal of Mathematical Economics 2, 21-33.

Mas-Colell, A. 1985. The Theory of General Economic Equilibrium: A Differentiable Approach. Cambridge: Cambridge University Press.

Radner, R. 1972. Existence of equilibrium of plans, prices, and price expectations in a sequence of markets. Econometrica 40, 289-303. 
Robertson, D. 1931. Wage grumbles. In Economic Fragments. London: P.H. King.

Samuelson, P. 1947. Foundations of Economic Analysis. Cambridge, MA: Harvard University Press.

Wieser, F. 1927. Social Economics. New York: Greenberg.

\section{fair allocation}

We survey the theory of equity in concretely specified economic environments. The literature concerns the existence of allocation rules satisfying various requirements of fairness expressed in terms of resources and opportunities understood in their physical sense (and not in terms of abstract entities such as utilities or functionings). For lack of space, we often give only representative references. Detailed treatments of the subject are Young (1994), Brams and Taylor (1996), Moulin (1995; 2003), and Thomson (1995b; 2006c).

\section{Concepts}

We introduce concepts central to the classical problem of fair division. These have much broader applicability, but for other models they sometimes have to be reformulated. Also, as models vary in their mathematical structures, the implications of a given concept may differ significantly from one to the other.

In an economy, there is a social endowment of resources to be distributed among a group of agents who are collectively entitled to them. For what we call a classical problem of fair division, the resources are infinitely divisible private goods, and preferences are continuous, usually monotonic (sometimes strictly so), and convex. In an economy with individual endowments, each agent starts out with a share of society's resources; the issue in this case is to redistribute endowments. In a generalized economy, some resources are initially owned collectively and others are individual endowments (Thomson, 1992; Dagan, 1995). A solution associates with each economy a non-empty subset of its set of feasible allocations. A rule is a single-valued solution.

An axiomatic study begins with the formulation of requirements on solutions (or rules). Their logical relations are clarified and their implications, when imposed in various combinations, are explored. For each combination of the requirements, do solutions exist that satisfy all of them? If the answer is 'yes', can one characterize the class of admissible solutions?

A punctual requirement applies to each economy separately. The main question then is the existence, for each economy in the domain under consideration, of allocations satisfying the requirement. First are bounds on welfares defined agent-by-agent, in an intra-personal way. Some are lower bounds, offering agents welfare guarantees. Others are upper bounds, specifying ceilings on their welfares. An allocation satisfies no-domination of, or by, equal division, if no agent receives a bundle that contains at least as much as an equal share of the social endowment of each good, and more than an equal share of the social endowment of at least one good, or a bundle that contains at most as much as an equal share of the social endowment of each good, and less than an equal share of the social endowment of at least one good (Thomson, 1995b). It satisfies the equal-division lower bound if each agent finds his bundle at least as desirable as equal division (Kolm, 1972; Pazner, 1977; and many others).

Second are requirements based on interpersonal comparisons of bundles, or more generally, 'opportunities', involving exchanges of, or other operations performed on, these objects. An allocation satisfies no domination across agents if no agent receives at least as much of all goods as, and more of at least one good than, some other agent (Thomson, 1983a). It satisfies no-envy if each agent finds his bundle at least as desirable as that of each other agent (Foley, 1967; Kolm, 1973, proposes a definition that encompasses many variants of the concept). The final definition is quite different in spirit: an allocation is egalitarian-equivalent if there is a reference bundle that each agent finds indifferent to his own bundle (Pazner and Schmeidler, 1978). Given a direction $r$ in commodity space, it is r-egalitarian-equivalent if it is egalitarianequivalent with a reference bundle proportional to $r$. Of particular interest is when $r$ is the social endowment.

A relational requirement prescribes how a rule should respond to changes in some parameter(s) of the economy. The idea of solidarity is central: if the environment changes, and whether or not the change is desirable, but no one in particular is responsible for the change, that is, no one deserves any credit or blame for it (or no one in a particular group of agents is responsible for the change,) the welfares of all agents (or all agents in this particular group), should be affected in the same direction: all 'relevant' agents should end up at least as well off as they were initially, or they should all end up at most as well off. In implementing this idea, the focus is usually on a particular parameter. When the parameter belongs to a space that has an order structure, as is frequent, one can speak of the parameter being given a 'greater' or 'smaller' value in that order. Then, together with efficiency, the solidarity idea often implies a specific direction in which welfares should be affected: when a Pareto improvement is possible, all relevant agents should end up at least as well off as they were initially; otherwise, all should end up at most as well off. Thus, solidarity takes the form of a 'monotonicity' requirement. Examples are resource monotonicity: if the social endowment increases, all agents should end up at least as well off as they were initially (Thomson, 1978; Roemer, 1986a; 1986b; Chun and Thomson, 1988); technology monotonicity, a similar requirement when technology expands (Roemer, 1986a; Moulin and Roemer, 1989); population monotonicity: if population expands, all agents initially present should 